
\title{
28 Research Square \\ Spontaneously Hypertensive Rat as a Model of Intrauterine Growth Restriction
}

Joana Asensio

CONICET Mendoza

Rodrigo D. García

CONICET: Consejo Nacional de Investigaciones Cientificas y Tecnicas

\section{Sergio G Benitez}

CONICET: Consejo Nacional de Investigaciones Cientificas y Tecnicas

\section{Susana R Valdez}

CONICET: Consejo Nacional de Investigaciones Cientificas y Tecnicas

Alicia Mabel Seltzer ( $\square$ aseltzer@fcm.uncu.edu.ar)

CONICET: Consejo Nacional de Investigaciones Cientificas y Tecnicas https://orcid.org/0000-00029903-7254

\section{Research Article}

Keywords: Glial Fibrillary Acidic Protein, Isolectin B4, Caveolin-1, Spontaneously Hypertensive Rat, IUGR/FGR

Posted Date: February 4th, 2022

DOI: https://doi.org/10.21203/rs.3.rs-1315270/v1

License: (c) (1) This work is licensed under a Creative Commons Attribution 4.0 International License.

Read Full License 


\section{Abstract}

Intrauterine growth restriction or foetal growth restriction (IUGR/FGR), is a condition associated to motor and cognitive deficits in which a foetus fails to achieve its genetically determined growth potential due to placental insufficiency. Spontaneously hypertensive rat (SHR) is a strain that endure pregnancy with placental insufficiency and their offspring are currently considered as an experimental model for ADHD and potentially for IUGR. Our study confirmed a delay in physical and neurological development, decreased brain genomic expression of IGF-1, alterations in cerebellar corticogenesis, foliation and Bergmann glia (fragmentation of GFAP labelling) during the immediate postnatal period. In the cerebellar cortex and white matter, using the isolectin B4, we observed the presence of microglial cells of amoeboid phenotype at P14. In most brain areas, including the meninges, we observed a marked number of dilated blood vessels. The cerebral cortex microvasculature analysis with CAV-1 presented a large index of Lacunarity and reduced expansion all through the brain parenchyma. On the basis of the present results, we reinforce the proposal of considering the pregnant SHR rat as a suitable experimental model of asymmetric IUGR and neurodevelopmental delay. The offspring of these rats display useful cellular and molecular markers for the monitoring of therapeutic interventions that could prevent or attenuate the disturbances initiated during the gestational stage.

\section{Highlights}

- SHR pups are proposed as a suitable experimental model for foetal growth impairment.

- Cerebellar development delay correlates with failure in the proper establishment of motor function.

- SHR present temporal delay in the maturation of the NVU and the presence of dilated blood vessels in the brain parenchyma.

\section{Introduction}

The adult SHR is an extensively studied paradigm modelling hypertensive disease, and it was developed by selectively breeding the Wistar strain of rats for high blood pressure [1]; [2]. One of the already known and most important features of SHR strain is that they endure pregnancy with placental insufficiency. Placental blood flow to the foetus is $26 \%$ reduced compared to the normotensive strain Wistar Kyoto (WKY), which is commonly used as a control [3]. SHR has been also proposed as a suitable animal model to study IUGR [4].

SHR foetuses grow under the circumstances of chronic oxygen and nutrient deprivation displaying typical circulatory changes, which in part represent adaptations to the suboptimal intrauterine environment. Chronic mild placental insufficiency can result in foetal growth restriction and deficits in neural connectivity and myelination [5].

Intra uterine growth restriction IUGR is a condition that affects $5-10 \%$ of pregnancies and is the second most common cause of perinatal mortality [6] and continues to be a leading cause of preventable 
stillbirth and poor neurodevelopmental outcomes in offspring, and furthermore is strongly associated with the obstetrical complications of iatrogenic preterm birth and pre-eclampsia [7]. Placenta-mediated IUGR/FGR occurs through chronic hypoxia owing to poor placental perfusion through a variety of mechanisms. Maternal vascular malperfusion is the most common placental disease contributing to IUGR [8]. Additionally, long standing epidemiological data support its association with chronic diseases such as hypertension and diabetes and seems to be a plausible link between in utero events and chronic circulatory diseases [9].

Both human and animal studies have demonstrated that the nature of the foetal neuropathology depends on many factors, and how the individuals resolve the severity of the insult depends on the efficiency of the auto regulatory mechanisms developed in both, the pregnant mother and in the offspring $[10 ; 11]$.

Rees et al. [5] discussed the complexity of the problem on establishing appropriate experimental models for perinatal brain damage and to date it remains difficult to study the underlying factors that cause IUGR/FGR in the way to prevent or attenuate the sequelae. In this work, we describe some neurodevelopmental landmarks of the SHR pups that may be suitable to test preventive and palliative putative therapeutic actions for the treatment of this condition. The aim of the present study was to underline the neurobehavioral profile during the postnatal development of SHR pups adding information to the observations reported by other authors and also to spot probable anatomical and morphological features of the nervous system that could be comparable to those present in the human suffering from IUGR. In the SHR rats we also focused on the cerebral vasculature, and of the development of the neurovascular unit. The characterization of more suitable animal models for the gestational pathologies is needed for the better understanding of underlying physio- pathological mechanisms and this will hopefully help to develop therapeutic strategies to protect the vulnerable foetuses and new-borns.

\section{Materials And Methods}

\section{Animals:}

Litters of Spontaneously Hypertensive (SHR) and age matched Wistar Kyoto (WKY, normotensive controls) were used for the experiments conducted in this work. Animals were maintained under $12 \mathrm{~h}$ light/dark cycle, controlled room temperature $\left(22 \pm 2^{\circ} \mathrm{C}\right)$ and free access to food and water. Systolic blood pressure was monitored in awake adult animals by means of plethysmography in the tail artery (CODA2 Non-invasive Blood Pressure System, Kent Scientific, Torrington, CT). Female pregnant were housed in steel cages with standard bedding and ad libitum access to food and water. Offspring birthdate was registered as postnatal day 0 (P0) and litter size was randomly reduced to 8 pups with the aim of reducing variability in weight or size during postnatal period [12].

Pups were weaned at P21 and reproductive parameters such as length of pregnancy, litter size and the number of stillbirths were registered. We also assessed sexual maturation and the onset of puberty by registering the day of vaginal opening [13] and testis descent [14; 15]. Animal housing, care and 
application of experimental procedures were conducted with minimal pain and suffering of the animals. The minimal number of animals to achieve statistically significant differences was determined according to the experimental design. For physical and neurological development assessment, three litters per group $(n=48)$ were used from birth to puberty. For morphometry studies, brain slices from both rat strains of the following postnatal ages: P7, P9, P14 and P21 were used. We allocated 6 animals per group and per age $(\mathrm{N}=48)$. For indirect immunofluorescence $(\mathrm{IFI})$ brain slices from three animals of each strain at P7 and P14 were processed, $\mathrm{N}=12$.

\section{Evaluation of postnatal development:}

Physical parameters, neurological reflexes and the development of motor coordination were evaluated in WKY and SHR rat pups applying standard tests at appropriate times between day of birth (P0) to puberty ( P40) (Supplementary Table 1). These observations were performed daily from 12.00 and 15.00 p.m. in an examination room with controlled environmental conditions of noise, lights and temperature.

Physical milestones: We evaluated the following physical parameters in rat pups from both strains: Body weight at birth (P0) was registered individually. Body growth, pups were weighted every other day from P2 to P36 and registered individually. Body weight gain was expressed in grams/day by using the following formula:

\section{Weight gain}

(weight (g) at P36-weight (g) at P2) /34 (days).

\section{Cephalization index (Cl)}

Each pup at P7 was weighed separately and the circumference of its head at the height of the snout and ears was measured. It was calculated as follows

$\mathrm{Cl}$ = head circumference $(\mathrm{cm}) /$ Body weight $(\mathrm{g})$.

Incisor eruption, the day both upper and lower incisors appeared was registered. Fur coat development, the day pups had their fur coat completed, including dorsal and ventral areas and fore and hind limbs was registered. Ear unfolding. bilateral ear detachment was observed from P0 until both outer ears were unfolded. Eye opening: the day both eyelids were completely separated, was registered.

\section{Neurological reflexes:}

Performance of neurological signs were assessed according to established methods [16]. A positive reflex response occurred when the pup was able to perform a task. The date of the first occurrence of the response was registered for further data processing.

Auditory startle: pups were exposed to a clapping sound and the first day of the startle response was registered as positive. Fore / hind limb grasp: fore and hind limbs were touched with a thin rod, and the 
first day of grasping onto the rod was recorded. Righting reflex. rats were placed on supine position on a flat surface and the time to perform the task was registered from the day of appearance. The maximal time was assigned if the pup was unable to perform the task within the allotted time (30 seconds). Each pup performed the test three times, with 5 minutes of rest between each trial; the mean was calculated for each animal. Negative geotaxis: pups were placed head downward on board $30 \mathrm{~cm}$ long and set at $45^{\circ}$; the board was covered with a tightly stretched non-slip mesh to ensure adequate friction. The first day they began to rotate $180^{\circ}$ with their nose pointing upwards was recorded and, from that moment on, the time in seconds to turn over was recorded. The maximal score was assigned if the rat was unable to perform the task within the allotted time (30 seconds). Each pup performed the test three times, with 5 minutes of rest between each trial; mean scoring was calculated for each animal.

\section{Motor coordination development:}

\section{Gait test}

The animals were placed in the centre of two concentric circles of 13 and $45 \mathrm{~cm}$ in diameter. The first day they began to move off the first circle was registered as walking initiation day. Time in seconds to move off both circles was recorded daily.

Foot-fault test (ladder rung walking test): this is a sensitive test for quantifying skilled locomotor movements [17]. The horizontal ladder consists of two transparent side walls and wooden rungs spaced at $2 \mathrm{~cm}$ intervals. Rats walk spontaneously from a neutral cage (starting location) to their home cage (goal) along a horizontal ladder. A camera was positioned at a slight ventral angle, so that both sides of the body could be recorded from a ventral view. Scoring was performed in terms of limb placement on a rung: a foot-fault occurs when a limb misses the rung followed by a deep or slight fall. In order to minimize variability, three trials were performed for each animal. The number of missteps and the time needed to cross the entire ladder in each trial were registered. The test was performed at 3, 4, 5 and 6 weeks of age.

Open field activity. Animals were observed for exploratory behaviour at 2, 3, 4, 5 and 6 weeks of age. After acclimatization to the environment, pups were placed into an activity / open field box consisting of $43 \mathrm{~cm}$ $x 42 \mathrm{~cm}$ with $20 \mathrm{~cm}$ high walls around. Pups were placed individually in the centre, always facing the same direction, and were video recorded for 5 minutes; the same testing box was used during the complete examination period. The following parameters were measured: head-lift, rearing (the act of standing onto the hind paws) and grooming (or self-cleaning) behaviour. Grooming behaviour included head washing, paw licking, body grooming, anogenital grooming and scratching [18].

\section{Histological analysis:}

\section{Tissue preparation}

Whole brains including the cerebellum were removed from transcardially perfused animals at different postnatal ages. The tissues were post-fixed overnight in Zamboni's fixative. The tissues were dehydrated 
in graded series of increasing concentration of alcohols, cleared in xylene and embedded in paraffin.

\section{Morphometric analysis}

Mid-sagittal slices of $8 \mu \mathrm{m}$-thick were obtained at the level of the vermis of the cerebellum (lateral 0 , Paxinos \& Watson Atlas, 7th ed. [19]), each containing a view of ten cerebellar folia. Paraffin was removed with xylene and histological sections were rehydrated in a series of decreasing concentration alcohols, then stained with cresyl-violet, and finally mounted with DPX. Images were captured using fixed acquisition settings with a conventional light microscope (Nikon 80i) attached to a digital camera. Cerebellar area and perimeter outline were carefully traced and measured using freehand selection at 40X magnification. Four images per animal were analyzed, and a total of 192 images were obtained and processed, corresponding to the 48 animals used in this study. Thickness of cerebellar cortical layer (EGL -external granule layer-, ML -molecular layer- and IGL -internal granular layer-) was measured using straight line selection in 200X magnification microphotographs. Preculminate (PCL), primary (PF) and secondary (SF) fissures were chosen for the measurement of layer thickness since these are the first fissures formed along embryological development [20]. Morphometric analysis was performed using Image $\mathrm{J}$ software (Image Processing and Analysis in Java; National Institutes of Health, USA). The results were expressed as mean \pm SEM of cerebellar area $\left(\mu \mathrm{m}^{2}\right)$, cerebellar perimeter $(\mu \mathrm{m})$ and thickness of cortical layers $(\mu \mathrm{m})$.

Indirect immunofluorescence (IFI) in free floating brain slices:

In order to study Bergmann's glia processes $30 \mu \mathrm{m}$-thick mid-sagittal sections containing the brain and the cerebellum were collected serially from both hemispheres. After re-hydration, the slides were immersed in TRIS buffer. Antigen retrieval was performed in $0.1 \mathrm{M}$ citrate buffer $(\mathrm{pH} 6)$ at $96^{\circ} \mathrm{C}$ during 15 min. Nonspecific binding was blocked with $1 \%$ BSA in TBS for 30 min at room temperature. Free floating sections were incubated overnight at $4^{\circ} \mathrm{C}$ with using the mouse anti-GFAP monoclonal primary antibody (1: 200, Cat. No. G3893, Sigma-Aldrich). After washes in TBS of 5 min each, sections were incubated for 2 $\mathrm{h}$ at room temperature with a secondary anti-mouse $\lg \mathrm{G}$ antibody coupled to fluorescein isothiocyanate (FITC) (1: 200, No. Fl- 2000, Vector, USA). To estimate non-specific binding and background fluorescence, sections were incubated omitting the primary antibody. Finally, sections were stained with DAPI (4',6Diamidine-2'-phenylindole dihydrochlorid, 10236276001 Roche, dilution 1:1000) overnight at $4^{\circ} \mathrm{C}$ and placed on gelatinized slides. Mowiol® 4-88 (Calbiochem \# 475904, Merck-Millipore) was used for mounting the coverslips under minimal lighting conditions. Image analysis: to study Bergmann's glia organization, images were acquired with an Olympus FluoView ${ }^{\mathrm{Tm}} \mathrm{FV}-1000$ confocal microscope (Olympus America Inc., Center Valley, USA) and processed with the FV 10-ASW 1.7 program (Olympus, Japan). 10 serial $X Y$ confocal images were acquired through $10 \mu \mathrm{m}$ thickness of each studied area, in.oib format and with a size of $800 \times 800$ pixels. They were then projected on the $Z$ axis, to obtain a $Z$ stack at 400X magnification. We drew an $8 \mu \mathrm{m}$ line perpendicular to the processes of Bergmann's glia and measured the number of intersections of these with the line was counted. The results were expressed as the mean linear density (processes / $\mu \mathrm{m}) \pm \mathrm{SEM}$. The individual processes were also measured 
throughout their entire length from the external granular layer (EGL) to the Purkinje Cell Layer (PC). Branched processes were measured to the longest end of the main projection; the lateral branches of the processes were not considered. Student's t test was applied to detect statistical differences; the results were expressed as length of the processes $(\mu \mathrm{m}) \pm$ SEM.

\section{Masson's trichrome stain}

A group of $8 \mu \mathrm{m}$ thick sagittal sections from each animal were processed following a previously published protocol [21].

Images were obtained with a conventional optical microscope (Nikon 80i) coupled to a digital camera (Sony) from the following brain areas: white matter of the cerebellum and pia mater of the cerebellar cortex, c. callosum, outer pyramidal layer of the primary motor cortex, hippocampus, thalamus and brainstem. Using the Image $\mathrm{J}$ program, the vessels were counted in a field of $1.2 \mathrm{~mm} 2$ (with the $10 \mathrm{X}$ objective) in the case of the white matter of the cerebellum and $0.288 \mathrm{~mm} 2$ (with the 20X objective) for the rest of the mentioned brain areas. Number of (dilated) vessels was expressed as Vessels $/ \mathrm{mm}^{2} \pm$ SEM. The data adjusted to a normal distribution for which the parametric Student $t$ test was applied for Statistical Analysis.

Neurovascular unit (NVU) assessment.

Sections were incubated with rabbit polyclonal anti-CAV-1 (1:200, SCBT, sc-894) and Far-red goat antirabbit IgG antibody (Vector Laboratories, Cat. No: DI-1649) for $2 \mathrm{~h}$ at room temperature. Endothelial cells in microvessels and microglia were assessed following the same procedure described above for free floating and indirect immunofluorescence using FITC-conjugated isolectin B4 from Bandeiraea simplicifolia (IB4, Vector Laboratories Cat\# B-1205, RRID: AB_2314661,1:400) on 30 um sagittal sections. Negative controls were obtained in the absence of primary antibody or the lectin. Finally, sections were mounted on glass slides with Mowiol 4-88 (Calbiochem \#475904). For the analysis of the vascular network we used AngioTool software [22].

Images of microglial cells were taken on an Olympus FV-1000 confocal microscope using a 100X objective. The images were captured and processed using the FV 10-ASW 1.7 program (Olympus, Japan). Three different areas per animal were analyzed within the white matter of the cerebellum. From each area, 10 optical planes in $Z$ of $1 \mu \mathrm{m}$ each were acquired; in oib format and $800 \times 800$ pixels that were projected into a stack $Z$, thus obtaining an $X Y Z$ image of each area for subsequent analysis. To quantitatively describe the morphology of the cells, the rounding index $(R I)$ was calculated as follows:

$R=4 \pi \mathrm{A} / \mathrm{P}^{\wedge} 2$

where A represents cell area $\left(\mu \mathrm{m}^{2}\right)$ and $\mathrm{P}$ cell perimeter $(\mu \mathrm{m})$ [23]. Image $\mathrm{J}$ program was used to measure $A$ and $P$ of each cell. RI was determined in 5 microglial cells per animal $(n=4)$. 


\section{Semi-quantitative RT-PCR:}

Rat pups were decapitated at P7 and P14 ( $n=4$ animals per group; $N=16$ total animals used), cerebellums were dissected and homogenized for RNA extraction using TRIzol ${ }^{\circledR}$ reagent (Ambion). Total RNA was extracted from whole cerebellums; the integrity and concentrations of the mRNA samples were determined with a spectrophotometer (Nanodrop, Thermo Scientific, Wilmington, DE, USA). cDNA was synthesized from $200 \mathrm{ng}$ total RNA using the M-MLV kit (Promega). IGF-1 and $\beta$-actin mRNA were detected using Taq polymerase (Firepol) with 35 and 30 cycles, respectively. We performed a negative control containing RNA instead of cDNA to rule out genomic DNA contamination.

Primers sequences were: IGF-1 Ec forward 5'-GACGCTCTTCAGTTCGTGTGTGGA-3'; reverse 5'GCAGGTGTTCCGATGTTTTGCAGG-3', predicted size 423 bp. For $\beta$-actin, forward 5'-

CGGAACCGCTCATTGCC-3' and reverse 5'-ACCCACACTGTGCCCATCTA-3', predicted size $289 \mathrm{bp}$. Both primers were custom-designed and checked using Primer-BLAST (https://blast.ncbi.nlm.nih.gov). Images of the RT-PCR SYBR Safe (Molecular Probes) stained agarose gels were acquired with an LAS-4000 system (Fujifilm) and quantified with Image $\mathrm{J}$ software.

\section{Statistical analysis:}

For comparisons between two single groups, parametric unpaired Student's t-test was applied using the D' Agostino Pearson normality test for assessing Gaussian distribution. ANOVA-1 and Multiple comparisons test was applied in the cases of comparison between strains in sets of two different ages. Two-way ANOVA and Multiple Comparisons post-test analysis was performed in the cases of comparison between strains in sets of several different ages. Results were expressed as mean \pm SEM Statistical processing was performed using Graph Pad Prism Software version 5.00 (GraphPad Software, San Diego California, USA). A significance level of $p<0.05$ was predetermined for all statistical analyses.

\section{Results}

\section{Physical milestones and neurological reflexes:}

Systolic blood pressure was monitored regularly in adult SHR rats that were not included in the experiments but were siblings and closely related to the experimental ones. The representative values are expressed as follows: WKY $124.17 \pm 9.09$ versus SHR $161.56 \pm 13 \mathrm{~mm}$ Hg Mean \pm SEM; $n=10$ animals per group. Reproductive performance was evaluated in terms of length of gestation, litter size, number of stillborn and puberty timing. For SHR gestation period was longer $(p<0.001)$ and litter size was significantly smaller comparing to WKY $(p<0.001)$. Stillborn were found among the SHR deliveries while they were absent in the WKY population (3:0 per litter). A significant delay in the onset of puberty was also observed for male and female pups $(p<0.0001)$.

Physical parameters assessed were body weight at birth, weight gain, cephalization index (Cl), completion of the fur coat, incisor eruption, ear unfolding and eye opening. SHR pups showed lower 
$(23 \%)$ weight at time of birth $(p<0.0001)$, gained less grams/day during the early postnatal period (P2P36) $(p<0.0001)$ and the $\mathrm{Cl}$ measured at P7 was significantly higher $(p<0.01)$ in comparison to WKY pups. This last observation indicates that a week after birth the SHR pups still maintain a disproportion between the size of the head and the rest of the body. Interestingly, the fur coat completed earlier and the incisors eruption showed a slight precocity in SHR pups $(p<0.05)$ although the ear unfolding and eye opening is significantly delayed in the SHR pups ( $p<0.0001$ and $p<0.001$ respectively). Regarding neurological reflexes, auditory startle showed a later onset in the SHR pups $(p<0.001)$, probably due to a delay in the opening of the auditory channel and, on the other side, no differences were observed in fore or hind limb grasps among strains, (Tables 1a and 1b).

Table 1

a. Physical characteristics and puberty initiation.

\begin{tabular}{|c|c|c|c|}
\hline & WKY & SHR & $p$ value \\
\hline Gestation length (days) & $20.86 \pm 0.14(7)$ & $21.86 \pm 0.14(11)$ & $\star \star \star ~$ \\
\hline Litter size (n) & $11.53 \pm 0.49(15)$ & $8.77 \pm 0.48(22)$ & $\star \star \star ~$ \\
\hline Weight at birth (g) & $6.93 \pm 0.26(29)$ & $5.32 \pm 0.10(30)$ & $\star * \star \star *$ \\
\hline Weight gain from P2-P36 (g) & $2,77 \pm 0,11(19)$ & $1,81 \pm 0,04(19)$ & 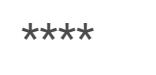 \\
\hline Cephalization index at P7 $(\mathrm{cm} / \mathrm{g})$ & $0.39 \pm 0.017(10)$ & $0.50 \pm 0.026(10)$ & ** \\
\hline Vaginal opening $(P)$ & $34.21 \pm 0.41(29)$ & $43.17 \pm 0.68(18)$ & $\star \star \star \star *$ \\
\hline Testicles descent $(P)$ & $20.62 \pm 0.14(13)$ & $24.39 \pm 0.70(13)$ & 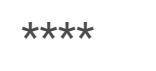 \\
\hline
\end{tabular}

Table 1

b. Average postnatal day of appearance of physical signs and neurological reflexes in WKY and SHR rats.

\begin{tabular}{|c|c|c|c|c|}
\hline & Sign & WKY & SHR & $p$ value \\
\hline \multirow[t]{4}{*}{ Physical milestones } & Incisor eruption $(P)$ & $10.81 \pm 0.20(31)$ & $10.24 \pm 0.18(25)$ & * \\
\hline & Fur coat completed(P) & $14.84 \pm 0.28(32)$ & $13.63 \pm 0.40(32)$ & * \\
\hline & Ear unfolding $(P)$ & $13.33 \pm 0.10(21)$ & $14.57 \pm 0.13(21)$ & $* * * *$ \\
\hline & Eye opening $(P)$ & $16.69 \pm 0.26(32)$ & $17.69 \pm 0.11(36)$ & $\star \star \star *$ \\
\hline \multirow[t]{3}{*}{ Neurological reflexes } & Auditory startle (P) & $12.90 \pm 0.53(20)$ & $13.96 \pm 0.99(22)$ & *** \\
\hline & Forelimb grasp (P) & $2.94 \pm 0.20(18)$ & $3.25 \pm 0.11(16)$ & ns \\
\hline & Hind limb grasp (P) & $3.54 \pm 0.35(18)$ & $3.97 \pm 0.28(16)$ & ns \\
\hline
\end{tabular}


Furthermore, we compared the body weight curves along the first postnatal month, and this analysis revealed significant lower values for the SHR pups noticed around P10 onwards, and these differences seemingly persisted up to P40 (WKY: $117.8 \pm 2.1$ (10); SHR: 85,4 \pm 3.2 (10), $p<0.0001$ Student's t test), (Fig. 1A).

Righting reflex and negative geotaxis were two tasks accomplished earlier by the SHR pups. For the righting reflex, statistically significant differences between the strains were noticed at P2 and extended for several days. After P8 the performance was even for the two strains (Fig. 1E.) For the negative geotaxis significant differences were present at all ages tested (from P20 to P26). (Fig. 1B). The walking initiation day was accomplished earlier by the SHR pups (WKY on day $15.60 \pm 0.66$ and SHR on day $12.12 \pm 0.53,(p<0.001)$ and in agreement, the gait test, was completed ahead of time by the SHR, reaching out the boundaries of both, the $13 \mathrm{~cm}$ and the $45 \mathrm{~cm}$ circles, before than their WKY counterparts (Figure $1 \mathrm{C}$ and D). In the foot-fault test, SHR pups accrued more mistakes than WKY at the 3rd and 4th week ( $p<0.05$; Figure 1F).

The animals were tested for motor and exploratory behaviour in an open-field arena and the number of head-lift, rearing and grooming behaviours were counted during a period of 5 minutes. SHR pups performed significantly lower number of head-lifts (3rd week: $p<0.01$; 4th and 5th weeks $p<0.05$ ), and lower rearing scores at the $3 \mathrm{rd}$ week, $(p<0.001)$. During the 4 th week the rearing activity was equivalent in both strains, but afterwards SHR reached significantly higher scores (5th week: $p<0.001$; 6th week: $p<0.0001$ (Figures 2A and 2B). On the contrary, grooming activity from weeks 3 to 5 was less frequent in the SHR pups (week 3rd and 5th : $p<0.01$ and week 4th : $p<0.001$, Figure 2C).

\section{Cerebellar morphometric analysis:}

We compared the size of sagittal slices stained with Cresyl Violet obtained from WKY and SHR pups at P7, P9, P14 and P21. Our results indicate that the differences between strains were significant at P9 $(p<0.05$ : cerebellar area) and ( $p<0.01$ : perimeter). These differences were attenuated by the second week of postnatal life (Fig. 3, C and D respectively). However, differences in the formation of fissures within the folia lasted up to later postnatal age, as it can be appreciated in Figure 3A3 and B3, the cerebellum of WKY P14 pups displayed deeper fissures within folia, while in SHR at the same age such folds are still incipient. (red arrows in Fig. 3A3, B3).

We also assessed the thickness of the cerebellar cortical layers at the level of the preculminate (PCF), primary $(P F)$ and secondary fissures $(S F)$ during postnatal development. (Figs. 4A and $C$ ). We observed that SHR, at the $P C F$ and $P F$ level presented a highly significant enlarged external granular layer (EGL) at $\mathrm{P} 14$ ( $p<0.0001$ vs WKY) and at P7, measured at the PCF level was also enlarged ( $p<0.0001$ vs WKY) but at the $P F$ level was less significant ( $p<0.01 v s$ WKY). At P14 EGL also was also enlarged ( $p<0.0001$ vs WKY) at the level of the SF. At P9, the difference was less significant in the $P F(p<0.05$ vs WKY) and at this same age, this difference was not observed in the PCF and SF (NS vs WKY at P9). The thickness of the molecular layer $(\mathrm{ML})$ increased during the second and third postnatal week in the WKY, but this 
increase occurred at a slower pace in the SHR (PCF: $p<0.01$ vs WKY at P14 and $p<0.001$ at P21; PF: $p<0.001$ at P14; SF: $p<0.05$ at P9, $p<0.0001$ at P14 and $p<0.001$ at P21). The thickness of the IGL only reached significant differences between strains in the PF at P21 (Fig. 4B). These results altogether indicate a delay in the SHR for the establishment of the final configuration of the cerebellar cortical layers.

Next we studied the Bergmann glial cells (BG). Cerebellar sagittal sections were stained with classical glial intermediate filament marker anti-GFAP antibody to visualize BG cytoskeleton. At P14 the cellular processes in the control strain were remarkably more organized with no interruptions, comparing to those of the SHR (Figs. 5A and $A^{\prime}$ and $B$ and $B^{\prime}$ ). The number of the main processes per $\mu$ m extending along the $\mathrm{ML}$ in these cells, showed no significant differences between strains, but their length was remarkably shorter ( $p<0.0001$ vs WKY) with numerous gaps in the trajectory, (Figs. 5C and D).

IGF-1:

IGF-1 mRNA expression was determined by RT-PCR in cerebellum of P7 and P14 animals. At the earlier age we observed slightly elevated levels of IGF-1 mRNA in the WKY, decaying by the end of the second week of life. In contrast, the SHR showed at P7 a lower expression of IGF-1 mRNA when compared to the normotensive strain $(p<0.05)$. By P14 the IGF-1 mRNA expression remained low in both rat strains, Figs. $6 \mathrm{~A}$ and $\mathrm{B}$.

\section{Brain vasculature:}

The trichrome Masson's stain did not reveal the presence of fibrotic connective tissue in the larger blood vessels brain tissue samples from both rat strains, but instead we noticed the presence of numerous vessels of enlarged luminal diameter in the cerebellar white matter cortex of the SHR at P14. ( $p<0.001$ vs WKY, Fig. 7A-F). These enlarged vessels were also observed in the cerebellar interlobular pia mater, the cerebral cortex, hippocampus, thalamus, corpus callosum and brainstem. (Supplementary Fig. 1). We assume that these vessels are consistent to low caliber arterioles and venules observed in the brain parenchyma and in the folds between the cerebellar folia near the pia mater.

\section{IB4 analysis:}

Isolectin B4 (IB4) specifically binds terminal a-galactosyl residues expressed by various cells including, but not limited to, endothelial cells but in general this tool is considered as a marker of the vascular elements [24].

AngioTool analysis applied in the cerebral cortex samples revealed, as expected, significant developmental increases in all vascular parameters: Vessels Percentage Area (VPA), Total Vessels Length (TVL), Vessels Area (VA), Total Number of Junctions (TNJ) and Total Number of End Points (TNEP) in the WKY strain, when comparing P14 vs. P7, (VPA: $p<0.0001$; TVL: $p<0.01$; VA: $p<0.01$; AVL: $p<0.05$; TNJ: $p<0.001$; TNEP: $p<0.05$ ) and a decrease in the Lacunarity index (Lac: $p<0.01)$. On the other side, the SHR 
strain showed only an increase in the vessels percentage area (VPA: $p<0.05)$ parameter, clearly indicating a delay in the vascular development with lower proliferation and in addition the Lac index in this case, did not show significance by the end of the second postnatal week, indicating a lack of territorial expansion, (Supplementary Fig. 2)

\section{CAV-1 immunostaining in cerebral cortex:}

We used Caveolin-1 antibody to identify microvessels associated to astrocytes and pericytes, the main components of the neurovascular unit (NVU). Figs. $8 \mathrm{~A}$ and $\mathrm{B}$ show the quantification of different vascular parameters applied to the collection of skeletonised images (by AngioTool), of the CAV- 1 labelled microvessels in the cerebral cortex (CTX) and cerebellum of WKY and SHR at P7 and P14. In the CTX of normotensive strain (WKY) these parameters increase significantly by the end of the second postnatal week (P14), (VPA: $p<0.0001$ vs P7; TVL: $p<0.001$ vs P7; VA: $p<0.01$ vs. P7; TNJ: $p<0.001$ vs. P7; TNEP: $p<0.0001 \mathrm{vs} P 7)$, indicating an intense spreading and ramification of the cerebral microvasculature during the early postnatal stage, and probably a more consolidated BBB. In contrast, the hypertensive strain (SHR) reached significant increases only in two of those parameters: VPA $(p<0.01)$ and TNEP $(p<0.05)$ when compared to the values obtained at the end of the first postnatal week (P7), indicating a probable delay in reaching a full functional NVU at that point of development (Fig. 8A). Notably, the Average Vessels Length (AVL) was not significantly different between strains or postnatal age, and the index of Lacunarity (LAC) decreased significantly in the normotensive strain (WKY: $p<0.0001$ ), indicating a rapid and efficient vascular coverage of the brain parenchyma by the end of the second postnatal week in the normotensive animals. In the hypertensive strain the decrease was more moderate (SHR: $p<0.01$ ), showing a weak vascular coverage of the brain cortex parenchyma, most probably affecting the brain cortex nutritional and metabolic demands, (Figs. 8C and D).

The same analysis in the cerebellum revealed a different view. Increases due to postnatal aging were smaller and statistically significant only in the following parameters: an increase in VPA ( $p<0.05$ vs P7) and a decrease in LAC $(p<0.01)$ vs P7) in the WKY strain, while the SHR strain remained at the same levels as those reached during the first postnatal age, although AVL seems to be slightly increased $(p<0.05$ vs P7) in this strain. The TNEP were stable at both ages and between strains, (Fig. 8B). The LAC index is decreased in the WKY strain $(p<0.01)$ in contrast, no significant differences were found in the SHR, indicating a delay in the microvascular coverage also observed in this SNC area (Fig. 8E and F.). These results are in concordance with the concept that the cerebral cortex matures at an earlier stage than the cerebellum, which is known to complete granular cells migration by P20 [25] and it is most likely that the development of the microvascular tree may follow the same pattern.

By using the double labelling CAV-1/ IB4 immunostaining were able to assess possible functional differences in the same brain area at P14. In the Cerebral Cortex it was possible to detect similar lower number of TNEP in the CAV-1 labelled elements in both strains (WKY: $p<0.01$ and SHR: $p<0.01$ ) comparing to the vessels labelled with IB4. We also observed differences among labels in the total vessels length parameter (TVL: $p<0.05)$ and a significant increase in LAC index $(p<0.05)$ in the SHRs, (Fig. 9C). The last mentioned difference can be clearly observed in the skeletonised images, as it is 
illustrated in Figs. 9A and B (a2-a4 and b2-b4). In the Cerebellum these differences between strains are absent supporting our previous observation of regional differences in the brain vascular maturation during the postnatal period, (Fig. 9D).

\section{Microglia:}

An additional element observed in this analysis was the abundant presence of round microglial cells mainly in the cerebellar white matter of the SHR, at both postnatal ages considered in this study, P7 and P14. These cells were identified using epifluorescence microscopy with the IB4 marker. which also labels microvessels in this tissue, as we mentioned before. In these images we were able to differentiate at least three morphological categories of these cells, representing different developmental stages. The stout microglial cells, showing thick processes were more predominant in the P7 samples of the normotensive rats, and turning to a more ramified phenotype at P14. The ameboid microglial cells, round and lacking processes, were present abundantly in the SHR samples at both P7 and persisted in P14, while they were scarcely found in the WKY almost disappearing by P14. The microglia with thin and long processes were observed more abundantly at P14, in the WKY however in the SHR these are seen intermingled with those of the ameboid phenotype, (Figs. 10A-D). The density of total microglia, regardless of morphology, obtained by adding up all three microglial morphologies described above, did not reach significant differences among groups (Fig. 10E). For a more detailed assessment and in order to settle the morphologic characteristics of the microglial cells present at P14, we used the confocal microscope images to calculate the Rounding Index. This analysis provided further information showing that the cerebellar white matter of SHR is populated by abundant small and round (ameboid) microglial cells, in contrast to the regular resident type microglia with elongated processes observed in the WKY at the same age in an equivalent brain area $(p<0.0001$ SHR vs WKY at P14, and $p<0.05$ SHR P14 vs SHR P7, Fig. 10F).

In the brain cortex of WKY, the total number of microglial cells was larger at P14 ( $p<0.01$ vs P7), and in the same trend an increase of smaller magnitude was observed in the SHR $(p<0.05$ vs $\mathrm{P} 7$, Supplementary Fig. 3).

\section{Discussion}

Neurodevelopment: In this work we assessed physical and neurodevelopmental parameters during the early life period of the SHR strain confirming previous observations from other groups describing alterations in the maturation of neurological reflexes and motor coordination deficits. Bassan et al [4] stated that the model of pregnant SHR closely simulate human IUGR caused by hypertension in pregnancy and should enable investigation of mechanisms of hypertension-mediated placenta-vascular injury. The SHR pups exhibited significant neurodevelopmental differences in the acquisition of neonatal reflexes (righting, negative geotaxis, gait) accomplishing the tasks at earlier postnatal age comparing to the pups of the control strain but, on the contrary present a delayed onset of puberty, also consistent with previous studies demonstrating that ovarian development, steroidogenesis, and responsiveness to 
gonadotropins are strongly compromised in these animals [13]. The precocious onset and restless responses observed in the righting reflexes, gait, negative geotaxis and the increases in foot faults observed in the SHR pups throughout the first weeks of life, may be linked to failure in the proper establishment and consolidation of the neuronal pathways involved in motor control. From their anxious phenotype, SHR strain has also been considered as an animal model of ADHD, since they 'naturally' display the main ADHD symptomatology such as hyperactivity and impulsivity [26], and in concordance, poor foetal growth increased the risk of ADHD in children [27; 28].

Increased foetal and neonatal mortality and morbidity as well as adult pathologic conditions are often associated to placental insufficiency [29; 30]. Pregnant Stroke Prone SHR displayed a significant reduction of placental mass and signs of placental insufficiency (i.e. hypertrophy and reduced branching morphogenesis of the labyrinth layer), associated with decreased offspring weights and increased cephalization index, which was also a common feature of the IUGR infants [31]. This response is associated to the physiological adaptation to preserve oxygen and nutrient supply to the brain (brainsparing phenomena) [32]. In the human IUGR foetuses due to placental insufficiency, the cerebellar to supratentorial volume ratio, calculated by MRI images, is affected [33]. In several species, rodents, guinea pig and sheep IUGR subjects are characterized by poor neuro-development and since the earlier studies of Altman [34] the experimental neurogenetic studies in the rat have established that all the cerebellar granule cells and a very high proportion of hippocampal granule cells are produced during the early postnatal period. The risk for behavioural disorders may be highest at late stages of foetal development, in prematurely born and small-for-age infants. Cerebellar lobule morphogenesis depends on conserved genetic mechanism to achieve the correct number of folds and also on local environmental clues, as for example, the Shh signal coming from the Purkinje cells (PC). Thus, while several malformations have a genetic basis, inflammation, fetal hemorrhage, and prematurity are often contributing factors [35]. In this study we did not include the assessment of the PC's because we did not observe anomalies in these cells using the routine laboratory stains. PC's anomalies were described in a previous work of our laboratory on P15 Wistar rat pups after acute postnatal hypoxia-ischemia. Disrupted BG processes labelled with vimentin, another cytoskeleton marker, were observed in the hypoxic-rat pups at P15 [36], but alternatively now we describe the apparent fragmentation of the BG processes when labelled with the GFAP glial marker. Sampson et al. [37], reported decreased foetal brain levels of glial fibrillary acidic protein (GFAP) and a-internexin during gestation in thyroidectomised animals, another condition affecting foetal growth and related to glial cell maturation. The apparent deficits in the intermediate filament scaffold of the SHR could be in correspondence to the delayed cerebellar foliation and a probable defect in the granular neurons migration from the EGL towards the IGL via the glial guidance inferred by the differences in the thickness of the cerebella cortical layers during postnatal development [38]. In addition, other rat models of IUGR also showed Bergmann glia disorganization at P7 and by P35, with a 10\% decrease in Bergmann glial fibre density [39]. We did not observe a decrease in the number of BG fibres and the granular neurones migration was not documented in this work. The observed fragmentation of the GB processed could be interpreted as a delayed exchange of the immature vimentin containing filaments to the more developed GFAP containing cellular processes, however, this issue still remains to be investigated. 
Recently it was described that Yap/Taz, two well-established downstream effectors of the Hippo pathway, known to regulate organ size by directing proliferation and apoptosis, and highly expressed in radial glia are playing a crucial role in establishing the radial scaffold and cellular polarity of neural progenitors during embryogenesis. Other effectors such as En2 [20], Jagged 1 [40], Atoh1 [41], Pard3a/JamC [42] have also been characterized among others, in mutant mice and preterm pigs delivered by $\mathrm{C}$-section, presenting cerebellar layering alterations. In further work it would be worth to explore the status of these effectors in the developing SHR in an attempt to explain the images shown here.

IGF-1: Insulin-like peptides in the brain participate in neurogenesis. The expression of IGF-1 is associated with regeneration and angiogenesis both in normal development and in pathological cases such as hypoxia. In the human a decrease of IGF-1 function, causes severe intrauterine growth restriction as well as microcephaly, sensorineural deafness, developmental delay, and metabolic abnormalities [43]. Under normal conditions the IGF-1 E2c variant predominates in cells differentiated into neurons. It expression decreases progressively in the first two postnatal weeks and increases under hypoxic conditions [44]. The parenchymal expression of IGF-1 and its splice variants can be particularly useful to assess the status of the SHR newborns. In the cerebellum of the SHR we observed a sustained low expression of the mRNA IGF-1Ec variant during the first and second postnatal weeks, in contrast to the WKY, which presented a small but significantly higher expression at P7. The delayed organ development and altered neurological parameters observed in the SHR together with a lower expression of this neurotrophic factor could be interpreted as a probable lower amount of differentiated neurons than the control strain, in the cerebellum at equivalent postnatal ages.

Brain microvasculature: Studies on the SHR microvasculature were mostly reported on the aging SHR brain [45]. Adult animals with established hypertension and "young animals", going as far back as 6-7 months old SHR and WKY are reported in the literature [46]. Previous morphometric analysis of capillary volume fraction, number of branches, capillary diameter and total length in adult SHRs with established hypertension concluded that those parameters were not significantly different among strains $[47,48]$. We applied the Masson trichrome stain to our samples of brain tissues from WKY and SHR at P14. This staining technique is usually used for assessing the presence of collagen fibres and fibrin. No signs of fibrosis or anomalous collagen deposits were observed in our very young SHR, before the initiation of hypertension. However, the stained sections for optic microscopy from both, white and grey matter and from the pial vasculature of SHR, revealed the presence of blood vessels, with diverse diameters, some of them remarkably dilated. Increased capillary diameter together with hyperplasia, abnormal shape and ultrastructure, changed cellular distribution of junctional proteins in the endothelial cells together with a decreased number of pericytes are considered as signs of transendothelial permeability, as it was described in PDGF-B/PDGFR-b knock out mice [49].

In an attempt to deepen the comparative analysis among strains of the brain capillaries we applied two recognized endothelial cells markers, CAV-1 and Isolectin B4 to the same tissue slices. CAV-1 is a membrane integral protein located to caveolae believed to play an important role in the regulation of multiple cellular processes, including cell growth, differentiation, endocytosis, cholesterol trafficking, and 
cellular senescence [50]. CAV-1 is thought to interact with occludin preventing the degradation of the structural proteins of the tight junctions (TJ) in the endothelial cells [51; 52]. On the other side, CAV-1 increases the BBB permeability via caveolae-based transcytosis and translocation of TJ protein. CAV-1 also inhibits matrix metalloproteinase-9 (MMP-9) which disrupts TJ proteins and basement membrane which means that CAV-1 may protect BBB integrity under certain circumstances, such as ischemic stroke. CAV-1 is also essential for NO-mediated and VEGF-induced angiogenesis, and there are evidences that Cav-1/VEGF-dependent pathway is important for neurogenesis, dendritic growth and arborisation [53].

In the human cerebral cortex CAV-1 is expressed by all the cell types that form the BBB, endothelial cells, pericytes, and vascular astrocytes [54]. A decrease in the expression of CAV-1 in the brain capillaries at early postnatal life, would imply a disruption of the mechanisms mentioned above, affecting the normal function of the NVU.

Bandeiraea simplicifolia as other lectins binds with high affinity to a-galactosyl residues of glycoproteins on the cell surface of the endothelium thereby allowing the visualization of blood vessel endothelial cells including endothelial phalanx, stalk and tip cells, as well as endothelial tip cell filopodia [55]. We analysed the expression of these two markers independently and in the combined form, and in this way we were able to observe differences among WKY and SHR in several parameters that characterize the brain cortex and cerebellar microvessels. These differences in general show a reduced amount of CAV-1 protein expression in the SHR's brain microvessels. On the contrary, IB4 labelling seems to run in parallel during the two first postnatal weeks, indicating the presence of an active process of angiogenesis sprouting and branching of the newly formed vessels in the process of forming the mature CNS vessel network in both rat strains.

AngioTool is commonly applied to assess the superficial plexus of the postnatal retina that forms a $2 \mathrm{D}$ flat vascular structure that can easily be assessed, imaged and analysed, in principle even with a standard fluorescence microscope. Keeping in mind this drawback, our comparative study between the hyper- and normotensive rat strains allowed us to detect a temporal delay in the maturation of the NVU in the SHR. This observation is in agreement to the rest of the developmental parameters that were assessed in this work. We interpret that the NVU plays an active role in the establishment of the neural circuits and proper synaptogenesis, and any anomaly in the consolidation of the BBB would affect many neural functions. However, we should consider cautiously this preliminary observation and a more thoughtful analysis is required, using confocal microscopy in carefully selected equivalent areas within particular brain regions.

An interesting result was the differential characteristics of the cerebellar and cerebral cortex microvasculature. The cortical development of the rat cerebellum was divided histologically into four stages: From birth to P4 occurs an intensive proliferation of neuroprogenitor cells in the EGL. By this time, cerebellar foliation has already begun. During the third stage (from P4 to P14), the neuroblasts, which had been produced in the EGL, undergo radial migration as stellate cells, basket cells and granular cells, the latter being the most predominant. The thickness of the EGL increased most prominently around P10. 
In the final stage, there is an almost complete disappearance of the EGL, resulting in the cellular architecture of the adult form of the cerebellar cortex [56]. Furthermore, these authors observed that vascular proliferation in the developing cerebellum correlated with EGL-formation pertains to the pial vessels, and not to the intraneural ones, which develop after neuronal cell migration has taken place, which would be around P21. This would explain the similar values we obtained in the microvessels analysis that were assessed before the consolidation of the cerebellar architecture, during the period of cell migration in both rat strains.

In the cerebral cortex, the comparative analysis between strains showed increased vessels length and Lacunarity index in the SHR when labelled with CAV-1 and no changes in the same vessels labelled with IB4, indicating that the microvasculature has developed in this organ, but may not have consolidated the structure of the NVU.

Microglia: Microglia in white matter regions displays unique characteristics in the first postnatal week of murine life. In both the corpus callosum and cerebellum microglia show amoeboid morphology and a similar transcription profile during development including high expression of genes related to priming of microglia, phagocytosis and migration at P7; characteristics which are already lost at P10. During the second and third week, microglia with both thick and thin processes are more abundant [57]. White matter microglia, perhaps by migration and phagocytosis, may play a special role in local tissue dynamics around P7, impacting oligodendrocyte homeostasis and myelination. This role is likely restricted to a brief window of time, after which cell numbers decline through apoptosis. As maturation continues into adulthood, resident microglial populations develop a common homeostatic role across white and grey matter regions [58]. Microglial cells are considered directly involved in the remodelling of neural synaptic circuits. The motor and sensorial stimulation during this developmental period may be regulating synaptic connections and microglial phagocytic activity in the cerebellum. By the second postnatal week rat pups generally open their eyes and also begin to respond to auditory signals [59], both neurological parameters are delayed by about one day in the SHR pups [60], and the presence of immature microglia at this time may correlate to the cerebellar morphological features described in this work.

Intrauterine growth restriction (IUGR) model of uterine arteries ligation cause delayed oligodendrocyte maturation and myelination accompanied by inflammatory responses in the rat [61]. Similar situation has been described in the IU growth restricted humans [62]. Under these circumstances, the placental insufficiency derives in chronic inflammatory processes, which may not be apparently the case of the SHR at this early postnatal period. However, around the time when hypertension signs begin to manifest, the underlying inflammatory factors may have been accumulating during an apparently silent early postnatal period. A more detailed study entailing inflammation markers assessed during the first and second postnatal weeks is still required to answer this issue.

The unfavourable uterine environment causing growth restriction results in programming that predisposes IUGR/FGR infants to long-term health issues such as poor physical growth, metabolic syndrome, cardiovascular disease, neurodevelopmental impairment and endocrine abnormalities [63]. To 
find reliable biological markers for the development and evaluation of early therapeutic interventions during gestation and early postnatal life remains as a complex and challenging objective. In sight of the present results we propose to consider the developing SHR as a relevant translational animal model for developmental impairment and preliminary results from our group are showing the possibility of reversal of the negative signs (unpublished results).

\section{Conclusions}

SHR pups exhibited significant neurodevelopmental differences in the acquisition of neonatal reflexes (righting, negative geotaxis, gait) accomplishing the tasks at earlier postnatal age, a delayed onset of puberty and higher difficulty in performing motor skills according to the results of the foot fault test. Their cerebellums were slower in completing the postnatal organogenesis, displaying differences in the thickness and delayed foliation. of the cortical layers. We also observed an apparent fragmentation of the BG processes when labelled with the GFAP glial marker and a sustained lower expression of the mRNA IGF-1Ec variant during the first and second postnatal week which could lead to a lower amount of differentiated neurons in this tissue. In several SHR brain areas we noticed the presence of dilated blood vessels. AngioTool analysis of the microvasculature in the cerebral cortex of this strain of rats revealed a reduced amount of CAV-1 protein expression in most of the parameters considered and an increased Lacunarity Index for this marker, and on the contrary no changes in the same vessels labelled with IB4, indicating that the microvasculature has developed in this organ, but it may have not consolidated the functional structure of the NVU. The IB4 marker also showed that the cerebellar white matter of SHR by the second postnatal week is still populated by abundant ameboid microglial cells, in contrast to the regular resident type microglia with elongated processes observed in the WKY.

We conclude that the neurodevelopmental characteristics of the SHR may constitute useful milestones to test possible preventive therapeutic approaches for IUGR and other perinatal events that interfere with normal growth and development.

\section{Abbreviations}

BBB: Blood Brain Barrier; CAV-1: Caveolin-1; GFAP: Glial Fibrillary Acidic Protein; IB4: Isolectin B4 (Bandeiraea simplicifolia); IGF-1: Insulin Growth Factor-1; IUGR/FGR: Intrauterine Growth Restriction/ Foetal Growth Restriction; NVU: Neurovascular Unit; SHR: Spontaneously Hypertensive Rat; WKY: Wistar Kyoto Rat.

\section{Declarations}

\section{Acknowledgements}

The SHR rats were bred and kindly provided by the Cátedra de Fisiopatología, Facultad de Ciencias Médicas de la Universidad Nacional de Cuyo (Mendoza- Argentina). The research fellowships to JA, SB 
and RG were granted by CONICET. We thank Dr. Cristian Acosta (IHEM-CONICET of Argentina) for the generous donation of IB4-FITC probe and for his useful comments during the elaboration of the manuscript. We also thank Dr. Sean Patterson (IHEM-CONICET of Argentina) for gently providing the antibody against CAV-1.

\section{Funding}

This research was funded by "Programa de Investigación, Desarrollo e Innovación. SECTYP-UNCuyo. Cód 06/P28. Período: 2015-2019" from Universidad Nacional de Cuyo, Mendoza-Argentina, granted to SV and AS and by a grant to SV from the "Agencia Nacional de Promoción de la Investigación y la Innovación, Ministerio de Ciencia, Tecnología e Innovación de Argentina" FONCYT-PICT 01762-2019.

\section{Author's contributions}

JA: Animal care and handling. Data acquisition and analysis for physical and neurodevelopmental tests. Sample preparation. Original Draft Writing and preparation of the manuscript. RG: Histological. Data Collection for vascular studies, AngioTool Analysis. SB: PCR Methodology, Data acquisition, Interpretation Analysis, Writing of the manuscript. SV: Review \& Editing of the manuscript, Visualization, Supervision, Funding Acquisition. AS: Conceptualization and Design, Methodology, Data analysis, Writing, Review \& Editing, General Supervision, Project Administration, Funding and resources acquisition. All authors read and approved the final manuscript.

Data Availability: The datasets generated during and/or analyzed during the current study are available from the corresponding author on reasonable request.

\section{Compliance with Ethical Standards:}

The animals used and experimental protocols were previously approved by our local Institutional Committee for Care and Use of Experimental Animals (CICUAL N ${ }^{\circ} 121 / 2017$ ) according to the National Institutes of Health Guide for the Care and Use of Laboratory Animals of the National Research Council (National Academies, U.S.A., $8^{\text {th }}$ Edition, 2011).

Code Availability: Not applicable.

Declarations of interest: We declare no conflict of interest.

Consent to participate: Not applicable.

Consent for publication: Not applicable.

\section{References}

1. Okamoto K, Aoki K (1963) Development of a strain of spontaneously hypertensive rats. Jpn Circ J 27:282-293. https:// doi.org/ 10.1253/jcj.27.282 
2. Saavedra JM (2009) Opportunities and limitations of genetic analysis of hypertensive rat strains. J Hypertens 27(6):1129-1133. https://doi.org/ 10.1097/HJH.0b013e32832bb832

3. Ahokas RA, Reynolds SL, Anderson GD, Lipshitz J (1987) Uteroplacental blood flow in the hypertensive, term pregnant, spontaneously hypertensive rat. Am J Obstet Gynecol Apr 156(4):10101015. . doi.org/ 10.1016/0002-9378(87)90378-4

4. Bassan H, Bassan M, Pinhasov A, Kariv N, Giladi E, Gozes I, Harel S (2005) The pregnant spontaneously hypertensive rat as a model of asymmetric intrauterine growth retardation and neurodevelopmental delay. Hypertens Pregnancy 24(3):201-211. . doi.org/ $10.1080 / 10641950500281142$

5. Rees S, Harding R, Walker D (2008) An adverse intrauterine environment: implications for injury and altered development of the brain. Int J Dev Neurosci 26(1):3-11. https:// doi.org/ 10.1016/j.ijdevneu.2007.08.020

6. Nardozza LM, Caetano AC, Zamarian AC, Mazzola JB, Silva CP, Marçal VM, Lobo TF, Peixoto AB, Araujo Júnior E (2017) Fetal growth restriction: current knowledge. Arch Gynecol Obstet 295(5):1061-1077. https:// doi.org/ 10.1007/s00404-017-4341-9

7. Audette MC, Kingdom JC (2018) Screening for fetal growth restriction and placental insufficiency. Semin Fetal Neonatal Med 23(2):119-125. . doi.org/ 10.1016/j.siny.2017.11.004

8. Zur RL, Kingdom JC, Parks WT, Hobson SR (2020) The Placental Basis of Fetal Growth Restriction. Obstet Gynecol Clin North Am 47(1):81-98. https://doi.org/ 10.1016/j.ogc.2019.10.008

9. Sehgal A, Murthi P, Dahlstrom JE (2019) Vascular changes in fetal growth restriction: clinical relevance and future therapeutics. J Perinatol 39(3):366-374. https://doi.org/ 10.1038/s41372-0180287-4

10. Peraçoli JC, Rudge MV, Sartori MS, da Silva Franco RJ (2001) Effects of hypertension on maternal adaptations to pregnancy: experimental study on spontaneously hypertensive rats. Sao Paulo Med J 119(2):54-58. https:// doi.org/10.1590/s1516-31802001000200003

11. Wlodek ME, Westcott KT, Ho PW, Serruto A, Di Nicolantonio R, Farrugia W, Moseley JM (2000) Reduced fetal, placental, and amniotic fluid PTHrP in the growth-restricted spontaneously hypertensive rat. Am J Physiol Regul Integr Comp Physiol 279(1):R31-R38. https://doi.org/ 10.1152/ajpregu.2000.279.1. R31

12. Agnish ND, Keller KA (1997) The rationale for Culling of Rodent Litters. Fundamental and applied toxicology 1997; 38: 2-6. doi.org/ 10.1006/faat.1997.2318

13. Pinilla L, Castellano JM, Romero M, Tena-Sempere M, Gaytán F, Aguilar E (2009) Delayed puberty in spontaneously hypertensive rats involves a primary ovarian failure independent of the hypothalamic KiSS-1/GPR54/GnRH system. Endocrinology 150(6):2889-2897. https:// doi.org/10.1210/en.20081381

14. Rodríguez-Padilla ML, de la Fuente M, Bellido C, Aguilar E, Aguilar R (1988) Desarrollo corporal y pubertad en ratas espontáneamente hipertensas [Body development and puberty in spontaneously hypertensive rats]. Rev Esp Fisiol 44(1):63-68 Spanish 
15. Amorim EM, Damasceno DC, Perobelli JE, Spadotto R, Fernandez CD, Volpato GT, Kempinas WD (2011) Short- and long-term reproductive effects of prenatal and lactational growth restriction caused by maternal diabetes in male rats. Reprod Biol Endocrinol ; 9:154. . doi.org/ 10.1186/14777827-9-154

16. Nguyen AT, Armstrong EA, Yager JY (2017) Neurodevelopmental Reflex Testing in Neonatal Rat Pups. J Vis Exp 24(122):55261 . doi.org/ 10.3791/55261

17. Metz GA, Whishaw IQ (2002) Cortical and subcortical lesions impair skilled walking in the ladder rung walking test: a new task to evaluate fore- and hindlimb stepping, placing, and coordination. $J$ Neurosci methods; 115:169-179. https:// doi.org/ 10.1016/s0165-0270(02)00012-2

18. van den Buuse M, de Jong W (1987) Grooming behavior of spontaneously hypertensive rats. Neurosci Lett 77(1):71-75. https://doi.org/ 10.1016/0304-3940(87)90609-4

19. Paxinos G, Watson C (2007) The Rat Brain in Stereotaxic Coordinates. Academic Press, San Diego

20. Sillitoe RV, Joyner AL (2007) Morphology, molecular codes, and circuitry produce the threedimensional complexity of the cerebellum. Annu Rev Cell Dev Biol 23:549-577.

10.1146/annurev.cellbio.23.090506.123237.. https://doi.org/ 10.1007/ 7854_2011_126

21. Rieppo L, Janssen L, Rahunen K, Lehenkari P, Finnilä MAJ, Saarakkala S (2019) Histochemical quantification of collagen content in articular cartilage. PLoS ONE 14(11):e0224839. https:// doi.org/ 10.1371/journal.pone.0224839

22. Zudaire E, Gambardella L, Kurcz C, Vermeren S (2011) A computational tool for quantitative analysis of vascular networks. PLoSOne 6(11):e27385. https://doi.org/ 10.1371/journal.pone.00273857

23. Sierra A, Navascués J, Cuadros MA, Calvente R, Martín-Oliva D, Ferrer-Martín RM, Martín-Estebané M, Carrasco MC, Marín-Teva JL (2014) Expression of inducible nitric oxide synthase (iNOS) in microglia of the developing quail retina. PLoS One, 29;9(8): e106048.

https://doi.org/10.1371/journal.pone.0106048

24. Lyons SA, Pastor A, Ohlemeyer C, Kann O, Wiegand F, Prass K, Knapp F, Kettenmann H, Dirnagl U (2000) Distinct physiologic properties of microglia and blood-borne cells in rat brain slices after permanent middle cerebral artery occlusion. J Cereb Blood Flow Metab 20(11):1537-1549. https:// doi.org/10.1097/00004647-200011000-00003

25. Rahimi-Balaei M, Bergen H, Kong J, Marzban H (2018) Neuronal Migration During Development of the Cerebellum. Front Cell Neurosci 12:484. https://doi.org/10.3389/fncel.2018.00484

26. Sagvolden T, Johansen EB (2012) Rat models of ADHD. Curr Top Behav Neurosci. 2012; 9:301-15. https://doi.org/ 10.1007/7854_2011_126

27. Sucksdorff M, Lehtonen L, Chudal R, Suominen A, Joelsson P, Gissler M, Sourander A (2015) Preterm Birth and Poor Fetal Growth as Risk Factors of Attention-Deficit/ Hyperactivity Disorder. Pediatrics 136(3):e599-608. https://doi.org/ 10.1542/peds.2015-1043

28. Chen J, Chen P, Bo T, Luo K (2016) Cognitive and Behavioral Outcomes of Intrauterine Growth Restriction School-Age Children. Pediatrics 137(4):e20153868. . doi.org/ 10.1542/peds.2015-3868 
29. Gagnon R (2003) Placental insufficiency and its consequences. Eur J Obstet Gynecol Reprod Biol 110(Suppl 1):S99-107. https:// doi.org/10.1016/s0301-2115(03)00179-9

30. Burton GJ, Jauniaux E (2018) Pathophysiology of placental-derived fetal growth restriction. Am J Obstet Gynecol 218(2S). https:// doi.org/ 10.1016/j.ajog.2017.11.577. S745-S761

31. Barrientos G, Pussetto M, Rose M, Staff AC, Blois SM, Toblli JE (2017) Defective trophoblast invasion underlies fetal growth restriction and preeclampsia-like symptoms in the stroke-prone spontaneously hypertensive rat. Mol Hum Reprod. 1;23(7):509-519. https:// doi.org/ 10.1093/molehr/gax024

32. Cohen E, Baerts W, van Bel F (2015) Brain-Sparing in Intrauterine Growth Restriction: Considerations for the Neonatologist. Neonatology 108:269-276. https:// doi.org/10.1159/. 000438451

33. Polat A, Barlow S, Ber R, Achiron R, Katorza E (2017) Volumetric MRI study of the intrauterine growth restriction fetal brain. Eur Radiol 27(5):2110-2118. https:// doi.org/ 10.1007/s00330-016-4502-4

34. Altman J (1987) Morphological and behavioral markers of environmentally induced retardation of brain development: an animal model. Environ Health Perspect 74:153-168. . doi.org/ 10.1289/ehp.8774153

35. Leto K, Arancillo M, Becker EB, Buffo A, Chiang C, Ding B, Dobyns WB, Dusart I, Haldipur P, Hatten ME, Hoshino M, Joyner AL, Kano M, Kilpatrick DL, Koibuchi N, Marino S, Martinez S, Millen KJ, Millner TO, Miyata T, Parmigiani E, Schilling K, Sekerková G, Sillitoe RV, Sotelo C, Uesaka N, Wefers A, Wingate RJ, Hawkes R (2016) Consensus Paper: Cerebellar Development, vol 15. Cerebellum., pp 789-828. 6. doi.org/ 10.1007/s12311-015-0724-2

36. Benitez SG, Castro AE, Patterson SI, Muñoz EM, Seltzer AM (2014) Hypoxic preconditioning differentially affects GABAergic and glutamatergic neuronal cells in the injured cerebellum of the neonatal rat. PLoS ONE 9(7):e102056. https:// doi.org/ 10.1371/journal.pone.0102056

37. Sampson D, Pickard MR, Sinha AK, Evans IM, Leonard AJ, Ekins RP (2000) Maternal thyroid status regulates the expression of neuronal and astrocytic cytoskeletal proteins in the fetal brain. $J$ Endocrinol 167(3):439-445. https://doi.org/ 10.1677/joe.0.1670439

38. Tolcos M, McDougall A, Shields A, Chung Y, O'Dowd R, Turnley A, Wallace M, Rees S (2018) Intrauterine Growth Restriction Affects Cerebellar Granule Cells in the Developing Guinea Pig Brain. Dev Neurosci 40(2):162-174. https://doi.org/ 10.1159/000487797

39. McDougall ARA, Wiradjaja V, Azhan A, Li A, Hale N, Wlodek ME, Hooper SB, Wallace MJ, Tolcos M (2017) Intrauterine Growth Restriction Alters the Postnatal Development of the Rat Cerebellum. Dev Neurosci. 2017;39(1-4):215-227. doi.org/ 10.1159/000470902

40. Weller M, Krautler N, Mantei N, Suter U, Taylor V (2006) Jagged1 ablation results in cerebellar granule cell migration defects and depletion of Bergmann glia. Dev Neurosci 28(1-2):70-80. https://doi.org/ $10.1159 / 000090754$

41. Iskusnykh IY, Buddington RK, Chizhikov VV (2018) Preterm birth disrupts cerebellar development by affecting granule cell proliferation program and Bergmann glia. Exp Neurol 306:209-221. https:// doi.org/ 10.1016/j.expneurol.2018.05.015 
42. Iskusnykh IY, Fattakhov N, Buddington RK, Chizhikov VV (2020) Intrauterine growth restriction compromises cerebellar development by affecting radial migration of granule cells via the JamC/Pard3a molecular pathway. Exp Neurol., 336: 113537. https:// doi.org/ 10.1016/j. expneurol. 2020.113537

43. Lupu F, Terwilliger JD, Lee K, Segre GV, Efstratiadis A (2001) Roles of growth hormone and insulin-like growth factor 1 in mouse postnatal growth. Dev Biol 229(1):141-162. . doi.org/ $10.1006 /$ dbio.2000.9975

44. Beresewicz M, Majewska M, Makarewicz D, Vayro S, Zabłocka B, Górecki DC (2010) Changes in the expression of insulin-like growth factor 1 variants in the postnatal brain development and in neonatal hypoxia-ischaemia. Int J Dev Neurosci 28(1):91-97. https:// doi.org/ 10.1016/j.ijdevneu.2009.09.002

45. Xue Y, Liu N, Zhang M, Ren X, Tang J, Fu J (2020) Concomitant enlargement of perivascular spaces and decrease in glymphatic transport in an animal model of cerebral small vessel disease. Brain Res Bull 161:78-83. https://doi.org/ 10.1016/j.brainresbull.2020.04.008

46. Gesztelyi G, Finnegan W, DeMaro JA, Wang JY, Chen JL, Fenstermacher J (1993) Parenchymal microvascular systems and cerebral atrophy in spontaneously hypertensive rats. Brain Res 21(2):249-257. https:// doi.org/ 10.1016/0006-8993(93)90510-t

47. Lin SZ, Sposito N, Pettersen S, Rybacki L, McKenna E, Pettigrew K, Fenstermacher J (1990) Cerebral capillary bed structure of normotensive and chronically hypertensive rats. Microvasc Res 40(3):341357. https:// doi.org/10.1016/0026-2862(90)90032-m

48. Naessens DMP, de Vos J, Van Bavel E, Bakker ENTP (2018) Blood-brain and blood-cerebrospinal fluid barrier permeability in spontaneously hypertensive rats. Fluids Barriers CNS. 24;15(1):26. https:// doi.org/ 10.1186/s12987-018-0112-7

49. Hellstrom M, Gerhardt H, Kalén M, Li X, Eriksson U, Wolburg H, Betsholtz C (2001) Lack of pericytes leads to endothelial hyperplasia and abnormal vascular morphogenesis. J Cell Biol 153(3):543-553. https:// doi.org/ 10.1083/jcb.153.3.543

50. Nguyen KCT, Cho KA (2017) Versatile functions of caveolin-1 in aging-related diseases. Chonnam Med J. 2017; 53: 28-36. doi.org/ 10.4068/cmj.2017.53.1.28

51. Jiao YQ, Huang P, Yan L, Sun K, Pan CS, Li Q, Fan JY, Ma Z, Han JY (2019) YangXue QingNao Wan, a Compound Chinese Medicine, Attenuates Cerebrovascular Hyperpermeability and Neuron Injury in Spontaneously Hypertensive Rat: Effect and Mechanism. Front Physiol 10:1246. . doi.org/ 10.3389/fphys.2019.01246

52. Gu Y, Dee CM, Shen J (2011) Interaction of free radicals, matrix metalloproteinases and caveolin-1 impacts blood-brain barrier permeability. Front Biosci (Schol Ed) 3:1216-1231. https:// doi.org/10.2741/222

53. Huang Q, Zhong W, Hu Z, Tang X (2018) A review of the role of cav-1 in neuropathology and neural recovery after ischemic stroke. J Neuroinflammation 15(1):348. https:// doi.org/ 10.1186/s12974018-1387-y 
54. Virgintino D, Robertson D, Errede M, Benagiano V, Tauer U, Roncali L, Bertossi M (2002) Expression of caveolin-1 in human brain microvessels. Neuroscience 115(1):145-152. https://doi.org/ 10.1016/s0306-4522(02)00374-3

55. Laitinen L (1987) Griffonia simplicifolia lectins bind specifically to endothelial cells and some epithelial cells in mouse tissues. Histochem J 19(4):225-234 https:// doi.org/1 s9,39,42,43. $0.1007 / B F 01680633$

56. Yoshida Y, Ikuta F, Watabe K, Nagata T (1985) Developmental microvascular architecture of the rat cerebellar cortex. Anat Embryol (Berl) 171(2):129-138. https://doi.org/ 10.1007/BF00341407

57. Perez-Pouchoulen M, VanRyzin JW, McCarthy MM (2015) Morphological and Phagocytic Profile of Microglia in the Developing Rat Cerebellum. eNeuro. 31;2(4): ENEURO.0036-15.2015. doi.org/ 10.1523/ENEURO.0036-15.2015

58. Staszewski O, Hagemeyer N (2019) Unique microglia expression profile in developing white matter. BMC Res Notes 12(1):367. https://doi.org/10.1186/s13104-019-4410-1

59. Babola TA, Li S, Gribizis A, Lee BJ, Issa JB, Wang HC, Crair MC, Bergles DE (2018) Homeostatic Control of Spontaneous Activity in the Developing Auditory System. Neuron. 8;99(3):511-524.e5. https:// doi.org/ 10.1016/j.neuron.2018.07.004

60. Ferguson SA, Gray EP, Cada AM (2003) Early behavioral development in the spontaneously hypertensive rat: a comparison with the Wistar-Kyoto and Sprague-Dawley strains. Behav Neurosci 117(2):263-270. . doi.org/ 10.1037/0735-7044.117.2.262

61. Zanno AE, Romer MA, Fox L, Golden T, Jaeckle-Santos L, Simmons RA, Grinspan JB (2019) Reducing Th2 inflammation through neutralizing IL-4 antibody rescues myelination in IUGR rat brain. $J$ Neurodev Disord 11(1):34. https://doi.org/ 10.1186/s11689-019-9297-6

62. Wixey JA, Chand KK, Colditz PB, Bjorkman ST (2017) Neuroinflammation in intrauterine growth restriction. Placenta 54:117-124. https://doi.org/ 10.1016/j.placenta.2016.11.012

63. Kesavan K, Devaskar SU (2019) Intrauterine Growth Restriction: Postnatal Monitoring and Outcomes. Pediatr Clin North Am 66(2):403-423. . doi.org/ 10.1016/j.pcl.2018.12.009

\section{Figures}


A.

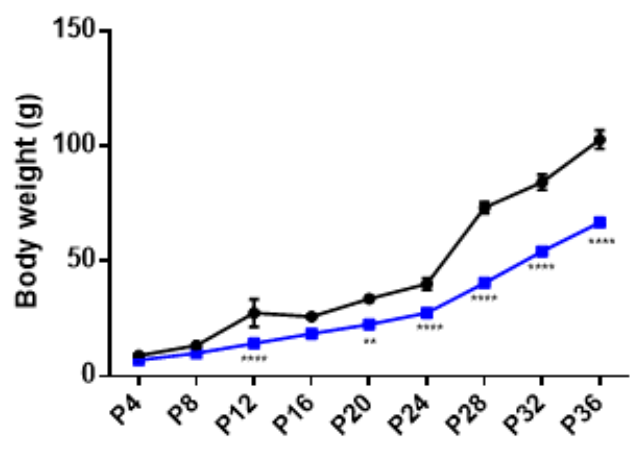

C.

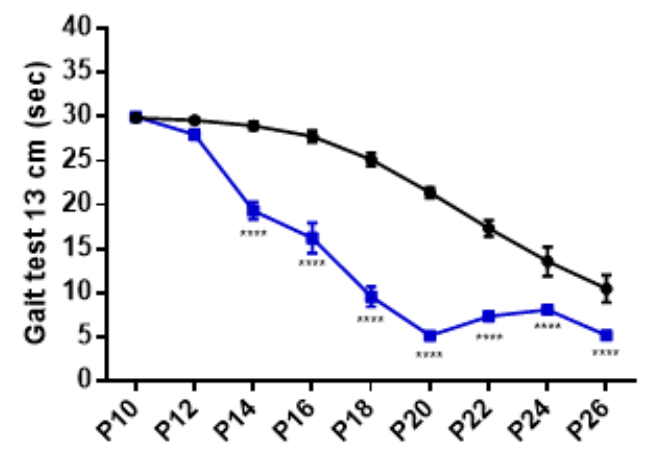

E.

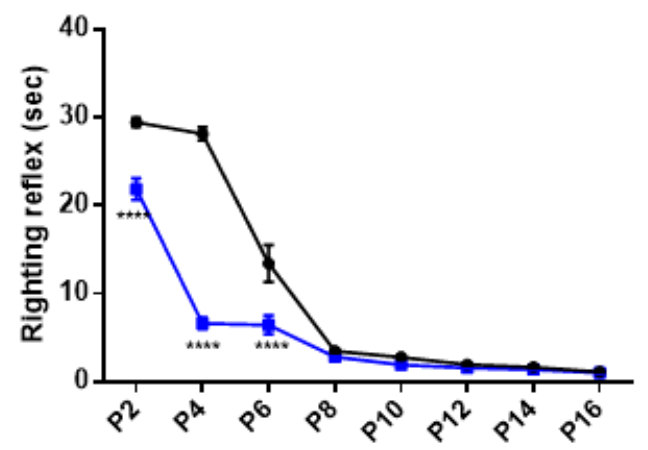

B.

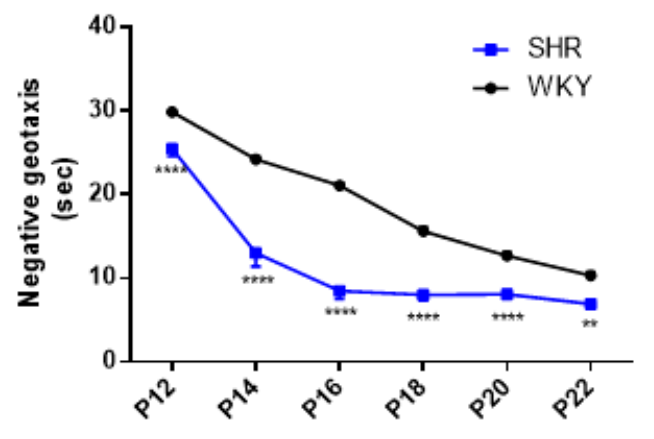

D.

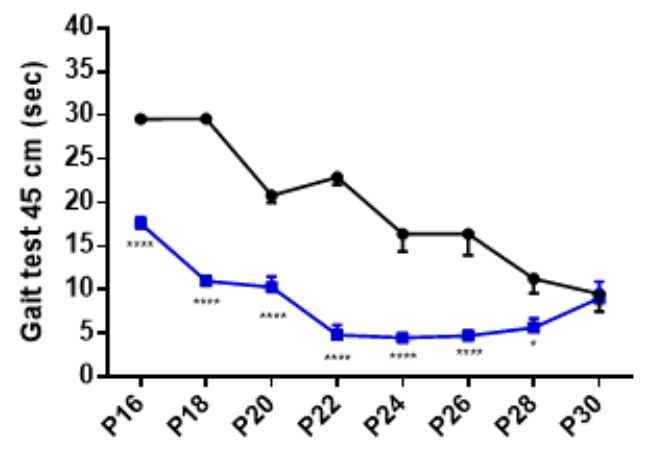

F.

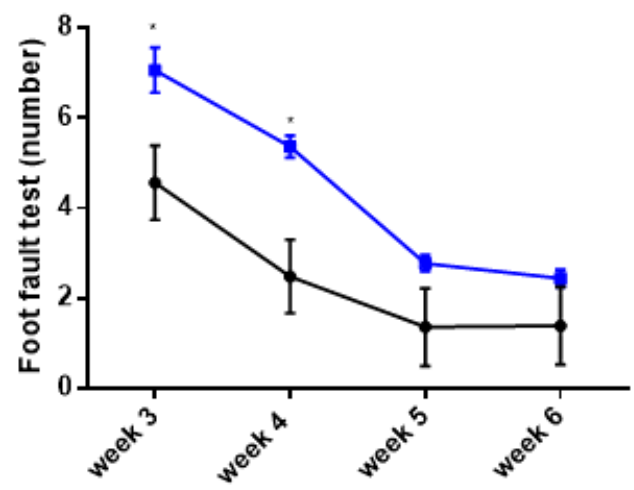

\section{Figure 1}

Comparative curves of development of body weight, neurological reflexes and motor functions between WKY and SHR strains. A.) Daily mean body weight; B.) Negative geotaxis test; C.) Gait test in $13 \mathrm{~cm}$ diameter circle; D.) Gait test in $45 \mathrm{~cm}$ diameter circle; E.) Righting reflex; F.) weekly performance in Foot-

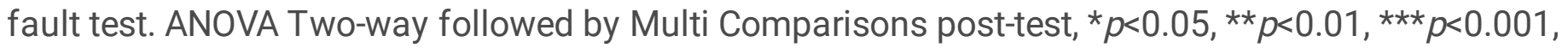
**** $p<0.0001$, SHR vs WKY. P(n): postnatal day. 
A.

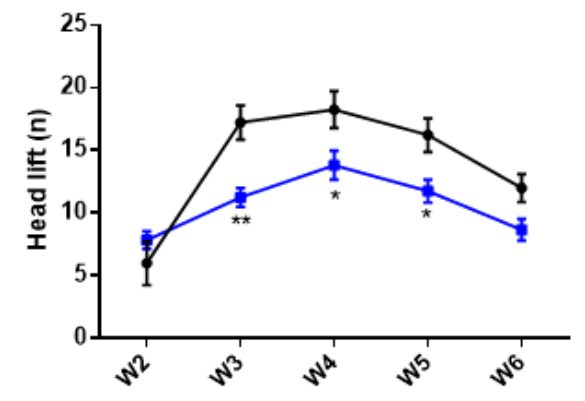

B.

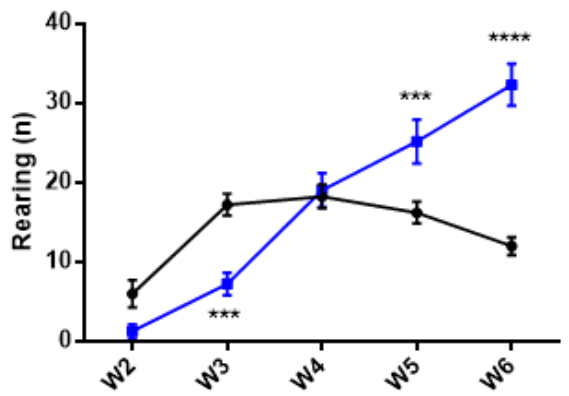

C.

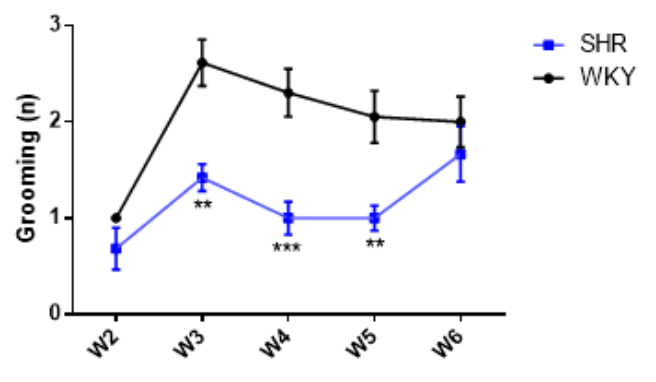

Figure 2

Weekly performance of locomotor activity during postnatal development. Open-field activity of WKY and SHR rats at 2, 3, 4, 5 and 6 weeks of age. A.) Head lift, B.) Rearing and C.) Grooming behaviour registered during 5 min sessions. ANOVA Two-way followed by Multiple Comparison test, ${ }^{\star \star \star \star} p<0.0001$; ${ }^{* \star \star} p<0.001$; $\star * x<0.01$ and ${ }^{*} p<0.05$ SHR vs. WKY. (W): week; (n): number of activities.

\section{Figure 3}

Morphometric analysis of the progression of area and perimeter of equivalent cerebellar slices during postnatal development. A.) and B.) Representative microphotographs (4X) of sagittal cerebellar slices stained with cresyl-violet from WKY (A1-A4) and SHR (B1-B4) pups at P7, P9, P14 and P21. Each individual image was adjusted to the red scale bar $=500 \mu \mathrm{m}$ shown at the bottom right. Red arrows indicate differences in the formation of fissures within the folia. C.) Quantitative analysis of cerebellar area and D.) perimeter at different postnatal ages to compare the evolution of these parameters between the two strains of rats. ${ }^{\star} p<0.05,{ }^{*} p<0.01$, SHR vs. WKY. ANOVA 2 Way and Multiple Comparisons Test, $\mathrm{n}=$ 3 - 6 animals per group. $\mathrm{P}(n)$ : postnatal day
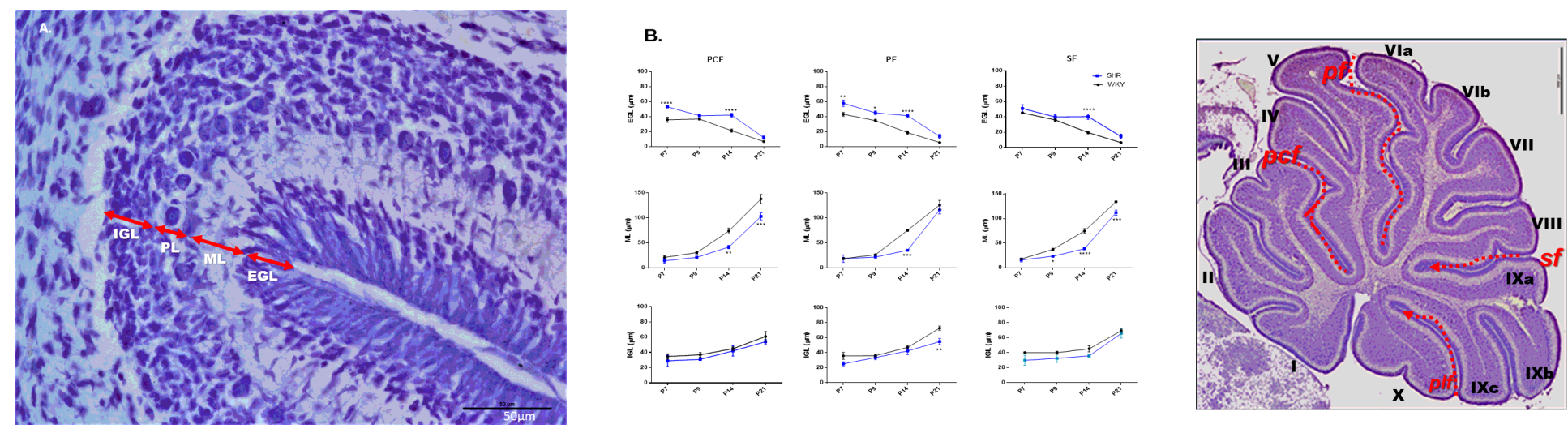

Figure 4 
Morphometric analysis of the thickness of cerebellar cortical layers of WKY and SHR rats during postnatal development. A.) Microscopic image (40X) of a representative cerebellar slice stained with cresyl-violet of a WKY rat at P9. The red arrows show the place of measurement of the different cerebellar cortical layers at the level of the PCF. EGL: external granular layer, ML: molecular layer, PL: Purkinje cell layer, IGL: internal granular layer. B.) Graphics represent the quantification of mean thickness $(\mu \mathrm{m}) \pm \mathrm{SEM}$ measured at the level of the preculminate fissure (PCF), primary fissure (PF) and secondary fissure (SF), at P7, P9, P14 and P21. ${ }^{\star} p<0.05,{ }^{*} p<0.01,{ }^{* \star *} p<0.001,{ }^{*} * \star * p<0.0001$ ANOVA 2 and Multiple comparisons test, $n=3-6$ animals per group. C.) The cerebellar fissures are indicated on a sagittal cerebellar slice of a WKY P14 (4X): pcf. preculminate fissure, pf: primary fissure; sf. secondary fissure; plf. posterolateral fissure. I-X: cerebellar lobules.

\section{Figure 5}

Bergmann glia in the cerebellar cortex. A.) Representative image (20X) of a cerebellar lobule from a WKY rat at P14 and B.) the equivalent image from a SHR P14 rat. Green epifluorescence corresponds to the GFAP labelling and blue to the DAPI stain. Scale bar $=100 \mu \mathrm{m}$. Discontinuously labelled BG processes are observed in the SHR. $A^{\prime}$.) and $B^{\prime}$.) are magnified images of the molecular layer seen in A. and B. using the confocal microscope $(60 \mathrm{X})$. Scale bar $=50 \mu \mathrm{m}$. C.) Mean \pm SEM of the number of processes per linear unit (processes $/ \mu \mathrm{m}$ ) and D.) Mean \pm SEM of the length of cellular processes $(\mu \mathrm{m})$, ${ }^{\star \star \star \star} p<0.0001, n=4$, animals per group (Unpaired Student's t test).

\section{Figure 6}

IGF-1 mRNA expression in cerebellum of WKY and SHR rat pups at P7 and P14. Representative PCR blot showing the expression of IGF-1 mRNA. A.) Each band represents a sample from one animal; $\beta$-actin gene expression was used as loading control; the ladder weight is indicated in bp number. In B.) Densitometry analysis averaging data from three separate assays. ${ }^{*} p<0.05$. ANOVA 1 way and Multiple comparisons test, $n=3-4$ animals per group.

\section{Figure 7}

Histological analysis of the microvasculature in the cerebellum and cerebral cortex. (A.F.) Representative photomicrographs from sagittal brain sections stained with Masson's trichrome. A.) shows a representative image of the cerebellar white matter (4X) of a WKY at P14; B.) equivalent image in a SHR at P14. Arrows in black outline highlight large diameter vessels. C.) an image (20X) of the cerebellar pia mater of a WKY P14; D.) same area in a SHR P14 showing a dilated vessel. E.) an image (40X) of the 
cerebral cortex of a WKY P14, F.) an equivalent section of a SHR P14. In G.) bars correspond to the mean \pm SEM of the number of dilated vessels / area unit in the cerebellar white matter, $\star \star \star *<<0.001, H$.) in the pia mater and I.) in the cerebral cortex (Unpaired Student's t test). WKY: $n=5 ;$ SHR: $n=4$ animals.

\section{Figure 8}

Caveolin-1 immunostaining of the microvasculature and quantitative analysis (using AngioTool) of several vascular parameters in the cerebral cortex (CTX) and cerebellum of WKY and SHR at P7 and P14. A.) Results of the comparative analysis between strains of the microvasculature of the brain cortex at the two developmental stages B.) Same analysis as in A. performed in the cerebellum. C). Quantitative analysis of the Lacunarity index in the CTX and E.) in the cerebellum. Representative images of the analyzed brain areas; D.) CTX; F.) Cerebellum. Scale bar $=50 \mu \mathrm{m} .{ }^{*} p<0.05 ;{ }^{\star \star} p<0.01 ;{ }^{* \star \star} p<0.001$ and $\star \star \star \star * p<0.0001$ ANOVA 1 and Multiple Comparisons test. $\mathrm{N}=3-5$ animals per group.

\section{Figure 9}

Comparative study of Caveolin-1/IB4 double-labelling in the cerebral microvasculature. In A.) representative images of the microvasculature using the far red filter for CAV-1 (a1) and the green filter for IB4 (a3); skeletonized images using AngioTool (a2 and a4) of the same view of the cerebral cortex of a WKY rat at P14. In B.) Representative images of the microvasculature using the far red filter for CAV-1 (b1) and the green filter for IB4 (b3); same images skeletonized using AngioTool (b2 and b4) of the cerebral cortex of a SHR rat at P14. Scale bar $=50 \mu \mathrm{m}$. C.) Quantitative analysis performed with AngioTool, of several vascular parameters of the microvessels in the cerebral cortex (CTX) and D.) in the cerebellum of WKY and SHR at P14. Bars in red colour represent the elements labelled with CAV-1 and bars in green colour the elements labelled with IB4. ${ }^{*} p<0.5$ and ${ }^{* *} p<0.01$ ANOVA 1 and Multiple comparisons test, $n=4-6$ animals per group.

\section{Figure 10}

IB4 labeling of microglial cells in the cerebellum of WKY and SHR at P7 and P14. A.) Representative image of a cerebellar lobule from a WKY rat at P7 showing the presence of microglial cells in the white matter; $A^{\prime}$.) enlarged image of a portion of $A$. B.) Representative image of an equivalent cerebellar region in a SHR at P7; $B^{\prime}$.) Image of $B$. seen at higher magnification; $C$.) and $C^{\prime}$.). Same as in (A.) and ( $A^{\prime}$.) but from a WKY rat at P14; D.) and $D^{\prime}$.) same as in (B.) and ( $B^{\prime}$.) but from a SHR at P14. Circles show microglial cells of different shapes and sizes (blue= ameboid; grey= stout; red= mature). Scale Bar 50 $\mu \mathrm{m}$. E.) Quantification of the total number of microglial cells of all shapes and sizes per area unit. F.) 
Quantification of the Rounding Index performed on confocal microscopic images of the same tissue samples. ${ }^{*} \mathrm{p}<0.05 ;{ }^{* \star \star \star} \mathrm{p}<0.0001$ ANOVA1 and Multiple comparisons test; $\mathrm{N}=4$ animals per group.

\section{Supplementary Files}

This is a list of supplementary files associated with this preprint. Click to download.

- SupplFig.2.tif

- SupplFig.1.tif

- SupplementaryTable.docx 